\title{
Epidemiology of pre-existing multimorbidity in pregnant women in the UK in 2018: a population-based cross-sectional study
}

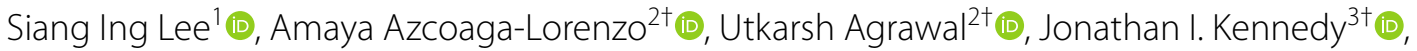

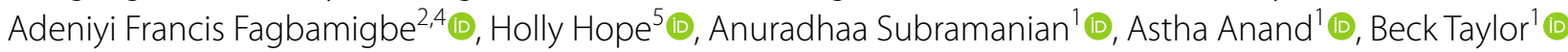

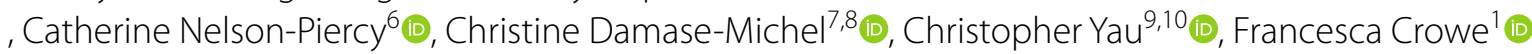

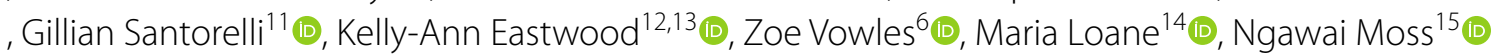

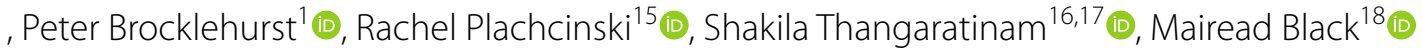

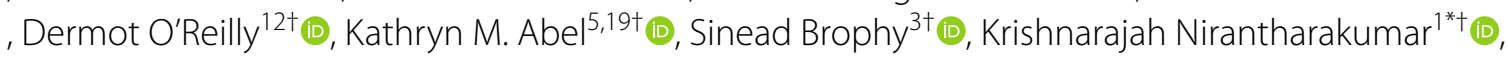
Colin McCowan ${ }^{2+}$ and on behalf of the MuM-PreDiCT Group

\begin{abstract}
Background: Although maternal death is rare in the United Kingdom, 90\% of these women had multiple health/ social problems. This study aims to estimate the prevalence of pre-existing multimorbidity (two or more long-term physical or mental health conditions) in pregnant women in the United Kingdom (England, Northern Ireland, Wales and Scotland).

Study design: Pregnant women aged 15-49years with a conception date 1/1/2018 to 31/12/2018 were included in this population-based cross-sectional study, using routine healthcare datasets from primary care: Clinical Practice Research Datalink (CPRD, United Kingdom, $n=37,641$ ) and Secure Anonymized Information Linkage databank (SAIL, Wales, $n=27,782$ ), and secondary care: Scottish Morbidity Records with linked community prescribing data (SMR, Tayside and Fife, $n=6099)$. Pre-existing multimorbidity preconception was defined from 79 long-term health conditions prioritised through a workshop with patient representatives and clinicians.

Results: The prevalence of multimorbidity was $44.2 \%$ (95\% Cl 43.7-44.7\%), 46.2\% (45.6-46.8\%) and 19.8\% (18.820.8\%) in CPRD, SAIL and SMR respectively. When limited to health conditions that were active in the year before pregnancy, the prevalence of multimorbidity was still high (24.2\% [23.8-24.6\%], 23.5\% [23.0-24.0\%] and 17.0\% [16.0 to $17.9 \%]$ in the respective datasets). Mental health conditions were highly prevalent and involved $70 \%$ of multimorbidity CPRD: multimorbidity with $\geq$ one mental health condition/s 31.3\% [30.8-31.8\%]).
\end{abstract}

\footnotetext{
*Correspondence: K.Nirantharan@bham.ac.uk

${ }^{\dagger}$ Amaya Azcoaga-Lorenzo, Utkarsh Agrawal and Jonathan I. Kennedy contributed equally to this work.

${ }^{\dagger}$ Dermot O'Reilly, Kathryn M. Abel, Sinead Brophy, Krishnarajah Nirantharakumar and Colin McCowan are joint senior authors. 1 Institute of Applied Health Research, IOEM Building, University of Birmingham, Edgbaston, Birmingham B15 2TT, UK

Full list of author information is available at the end of the article
}

(C) The Author(s) 2022. Open Access This article is licensed under a Creative Commons Attribution 4.0 International License, which permits use, sharing, adaptation, distribution and reproduction in any medium or format, as long as you give appropriate credit to the original author(s) and the source, provide a link to the Creative Commons licence, and indicate if changes were made. The images or other third party material in this article are included in the article's Creative Commons licence, unless indicated otherwise in a credit line to the material. If material is not included in the article's Creative Commons licence and your intended use is not permitted by statutory regulation or exceeds the permitted use, you will need to obtain permission directly from the copyright holder. To view a copy of this licence, visit http://creativecommons.org/licenses/by/4.0/. The Creative Commons Public Domain Dedication waiver (http://creativeco mmons.org/publicdomain/zero/1.0/) applies to the data made available in this article, unless otherwise stated in a credit line to the data. 
After adjusting for age, ethnicity, gravidity, index of multiple deprivation, body mass index and smoking, logistic regression showed that pregnant women with multimorbidity were more likely to be older (CPRD England, adjusted OR 1.81 [95\% Cl 1.04-3.17] 45-49years vs 15-19years), multigravid (1.68 [1.50-1.89] gravidity $\geq$ five vs one), have raised body mass index (1.59 [1.44-1.76], body mass index 30+ vs body mass index 18.5-24.9) and smoked preconception (1.61 [1.46-1.77) vs non-smoker).

Conclusion: Multimorbidity is prevalent in pregnant women in the United Kingdom, they are more likely to be older, multigravid, have raised body mass index and smoked preconception. Secondary care and community prescribing dataset may only capture the severe spectrum of health conditions. Research is needed urgently to quantify the consequences of maternal multimorbidity for both mothers and children.

Keywords: Multimorbidity, Multiple chronic conditions, Multiple long-term conditions, Pregnancy, Maternity, Epidemiology

\section{Background}

Multimorbidity is having two or more long-term health conditions [1]. Although well studied in other disease area, there is currently sparse literature for multimorbidity in pregnant women. Pregnant women with multimorbidity are at increased risk of adverse outcomes for mother and child [2,3]. Although maternal death is rare in the United Kingdom (UK), $90 \%$ of women who died during/within a year after pregnancy had multiple health and social problems [4].

Multimorbidity increases health care burden for patients, for instance, needing to attend multiple health care appointments and being on multiple medications [1]. These challenges increase during pregnancy, with the addition of specialist antenatal clinic appointments and monitoring, and concerns regarding how medications may affect the developing foetus.

Despite this, there is a dearth of basic information on the prevalence and types of pre-existing health conditions affecting pregnant women. Better understanding of the epidemiology of multimorbidity in pregnant women could help policy makers and health care providers plan services to prevent women from developing multimorbidity, for early detection and optimal management of health conditions prior to conception, and tailor maternity services to pregnant women with multimorbidity.

In the UK, most people are registered with a general practitioner (GP), the gatekeepers to primary care and specialist referrals. In secondary care, health care utilization administrative data are recorded for reimbursement. Thus, both provide good data sources for multimorbidity and pregnancy research.

This study aims to describe the epidemiology of preexisting multimorbidity in pregnant women. It also seeks to understand the utility of routine health care datasets in the study of multimorbidity in pregnant women, by using three datasets from different health care settings and across the four UK nations (England, Northern Ireland, Scotland and Wales).

\section{Methods}

\section{Study design and study period}

This was a cross sectional analysis of the prevalence of pre-existing multimorbidity prior to the start of pregnancy in the UK across three separate databases. We included index pregnancies where the conception date was between $1 / 1 / 2018$ and 31/12/2018.

\section{Inclusion and exclusion criteria}

Women aged 15-49years with a conception date in 2018 were eligible. Last menstrual period or gestational day 0 was considered the conception date [5]. When a woman had more than one pregnancy episode in 2018, the first recorded pregnancy in that year was included (not necessarily the first ever pregnancy). Women whose data did not meet standard quality checks were excluded (Additional file 1).

\section{Data sources}

This study used three datasets from different health settings, covering all four nations in the UK: Clinical Practice Research Datalink, (CPRD, England, Northern Ireland, Scotland and Wales), Secure Anonymized Information Linkage (SAIL, Wales) and Scottish Morbidity Records (SMR, Scotland).

\section{Primary care}

CPRD GOLD contains anonymized, longitudinal medical records for over 19 million patients in the UK (England, Northern Ireland, Scotland and Wales) from over 940 participating general practices; it currently covers $4 \%$ of UK GP practices and is widely acknowledged to be representative of the UK population [6]. It includes data on demographics, diagnoses and prescriptions [6]. Linkage to area based deprivation index was available for patients in England. Within CPRD GOLD, the CPRD Pregnancy Register is an algorithm that takes information from maternity, antenatal and delivery health records to detect pregnancy episodes and their outcomes [5]. 
The SAIL databank is a whole population level database in Wales. It is a repository of anonymized health and socio-economic administrative data and provides linkage at an individual level [7]. It holds data for 4.8 million people and covers $80 \%$ of Welsh GP practices [7]. Within SAIL, the National Community Child Health Dataset was used to detect pregnancies and was linked to the Welsh Longitudinal General Practice dataset and the Welsh Demographic Service dataset for diagnoses, prescriptions and demographics data respectively.

\section{Secondary care and community prescriptions}

SMR data was available from two Scottish regional health boards: National Health Service (NHS) Tayside and NHS Fife [8]. A dataset was created linking the Scottish Maternity Records (SMR02) to data from Hospital Admissions (SMR01), Mental Health Inpatients (SMR04), Accident and Emergency, and the Demography and Death registry. This covered diagnoses and demographic data for all inpatient stays and day cases for residents in the two regions. The dataset was also linked to the Prescribing Information System for data on all medications dispensed in the community. Pregnancies were detected from maternity records or pregnancy-related hospital admissions.

\section{Definition of multimorbidity}

Multimorbidity was defined by the presence of two or more pre-existing long-term physical or mental health conditions prior to the index pregnancy. We defined long-term conditions as conditions that have ongoing significant impact on patients, including conditions that are relapsing and remitting in nature.

One of the wider research aims is to mitigate the effect of multimorbidity on adverse pregnancy outcomes. As pregnancy related conditions (e.g., gestational diabetes and pregnancy induced hypertension) will be subsequently studied as maternal outcomes, they were not included in the definition of pre-existing multimorbidity.

An exhaustive list of long-term health conditions was first identified from existing literature $[4,9,10]$, in particular based on the work commissioned by Health Data Research UK on multimorbidity conceptualization [10] and health conditions that were leading indirect cause of death in the UK maternal mortality report (MBRRACE) [4]. This list and phenome definitions were refined and harmonized through workshops with our research advisory group, consisting of patient and public representatives, clinicians from general practice, obstetrics, maternal medicine, psychiatry, public health, and data scientists. Seventy nine health conditions were selected on the following basis: (i) prevalence; (ii) potential to impact on pregnancy outcomes; (iii) considered important by women; and (iv) recorded in the study datasets.

Diagnoses of these 79 long-term health conditions were determined from Read Codes version 2 (primary care datasets) and the International Classification of Disease 10th version (secondary care datasets) [11]. The validity of diagnostic coding has previously been shown to be good in primary care records and generally health conditions under payment for performance schemes, such as Quality Outcomes Framework, are well coded [12]. Code lists and phenome definitions used are available in Additional files 2 and 3.

\section{Data analysis}

The primary analysis was the prevalence of pre-existing multimorbidity in pregnant women. The denominator was the total number of index pregnancies identified in 2018, regardless of the pregnancy outcome. Additional analysis was performed for multimorbidity with at least one mental health conditions and active multimorbidity. Active multimorbidity limits common transient/episodic conditions (e.g., mental health, dermatological and atopic conditions and headaches) to those that were active in the 12 months preceding index pregnancy (Additional file 3).

Multivariable logistic regression was performed to examine the association of multimorbidity with maternal age (five-yearly categories), ethnicity, deprivation quintiles (patient level Index of Multiple Deprivation [IMD] for all three datasets), latest maternal pre-pregnancy body mass index (BMI) categories, latest pre-pregnancy smoking status, and gravidity (total number of pregnancies up to and including index pregnancy). Obesity was considered a covariate (BMI categories) instead of a health condition. For CPRD, practice level IMD was available for all four nations, but patient level IMD was only available for England, therefore, the regression analysis was limited to England. We then described the prevalence of individual health conditions, and the prevalence of mutually exclusive multimorbidity combinations.

Missing data were assigned to separate categories and included in the analyses. Sensitivity analysis was performed for CPRD (England), where missing ethnicity was imputed with data from linked hospital administrative data, and missing patient level IMD was imputed with practice level IMD.

Study results were presented for each dataset separately. Data were not combined as there was a possibility of patient overlap between CPRD (Wales, Scotland) with both SAIL (Wales) and SMR (Scotland). Deduplication was not possible as the datasets are anonymized, and only aggregated data were exported within the permission of the data access approval. 


\section{Post hoc analysis}

As our study found no association of recorded multimorbidity with social deprivation, we conducted a post hoc analysis in the CPRD cohort, with the list of conditions used to define multimorbidity in a seminal paper that found this association [13]. We also examined the association of selected health conditions with deprivation and ethnicity. Guided by our patient representatives, we analysed the prevalence of multimorbidity for selected health conditions to illustrate the burden of multimorbidity. The selected health conditions were: i) the top ten most common individual health conditions in this study, and ii) leading causes of maternal deaths [4].

Analysis was performed using STATA 16 and R. The study is reported in accordance with the RECORD guideline (Additional file 4).

\section{Results}

\section{Study population}

Overall, there were 37,641 (CPRD), 27,782 (SAIL) and 6099 (SMR) pregnant women aged 15-49 years included in the analysis in 2018. Additional Fig. 1 presents the flow chart for the study population selection. The characteristics of the study cohort is presented in Table 1 and Additional Table 1. Most of the study participants were 20-34 years old, of White ethnicity, of normal weight or were overweight pre-pregnancy and were non-smoker pre-pregnancy. Linkage to area-based data for patient level IMD was available for $75 \%$ of the CPRD study cohort in England. There were more affluent women in the primary care dataset but vice versa for SMR.

\section{Multimorbidity}

The prevalence of pre-existing multimorbidity in pregnant women was $44.2 \%$ (95\% confidence intervals [CI] 43.7 to $44.7 \%$ ), $46.2 \%$ (45.6 to $46.8 \%$ ) in CPRD and SAIL respectively (primary care dataset) but was halved in SMR's secondary care and community prescription dataset, $19.8 \%$ (18.8 to $20.8 \%$ ).

Over $70 \%$ of pregnant women with multimorbidity had mental health condition/s: $31.3 \%$ (30.8 to $31.8 \%$ ), $33.7 \%$ (33.1 to $34.2 \%$ ) and $14.6 \%$ (16.0 to $17.9 \%$ ) of pregnant women had multimorbidity with at least one mental health conditions in CPRD, SAIL and SMR respectively. The prevalence of active multimorbidity was half that of the primary analysis in primary care datasets, $24.2 \%(23.8$ to $24.6 \%$ ) and $23.5 \%$ (23.0 to $24.0 \%$ ) for CPRD and SAIL respectively, but remained similar for SMR, 17.0\% (16.0 to $17.9 \%)$. The percentage of pregnant women by the total morbidity count is available in Additional Table 2.
Characteristics associated with multimorbidity

The prevalence of pre-existing multimorbidity by the characteristics of pregnant women is presented in Additional Table 3. In the CPRD England study cohort $(n=13,075)$, when all characteristics were adjusted for, increasing maternal age and gravidity remained significantly associated with multimorbidity (maternal age $45-49$ years, adjusted odds ratio [aOR] $1.8,95 \%$ CI 1.0 to 3.2 ; gravidity $\geq 5,1.7,1.5$ to 1.9 ); pregnant women with BMI 25 to 29.9 (aOR 1.2, 95\% CI 1.1 to 1.3 ), BMI $30+(1.6,1.4$ to 1.8$)$, were smokers $(1.6,1.5$ to 1.8$)$ or exsmokers $(1.4,1.3$ to 1.6$)$ had higher odds of multimorbidity. However, higher odds of multimorbidity were not observed in pregnant women of ethnic minority groups or from more deprived socioeconomic groups (Table 2). Findings were similar in the sensitivity analysis of CPRD (England) using imputed data for missing ethnicity and IMD (Additional Table 4). In SAIL, the effect sizes of characteristics were generally similar to that in CPRD (England).

In SMR, after adjusting for all characteristics, higher odds of multimorbidity were observed only in those age 20-24 and 25-29years, had gravidity of $3+$, BMI $30+$, were smokers and ex-smokers and those from more deprived socioeconomic groups. The odds of multimorbidity were not higher in ethnic minority groups (Table 2).

\section{Post hoc analysis}

Post hoc analysis was performed to explore whether the lack of association of multimorbidity with deprivation in our primary care datasets was, in part, due to the health conditions we used to define multimorbidity. Logistic regression was repeated in CPRD (England) with the list of 31 health conditions used to define multimorbidity in Barnet et al's seminal paper [13], the adjusted variables were added in a step-wise manner. After adjusting for maternal age, ethnicity and gravidity, increasing levels of deprivation were associated with higher odds of multimorbidity (most deprived quintile aOR 1.30, 95\% CI 1.08 to 1.57 ). This association was attenuated and was no longer significant when raised BMI and smoking status were added to the model (aOR 1.05, 0.87 to 1.27, Additional Table 5, Fig. 1).

To test this hypothesis further, we repeated the logistic regression in the CPRD (England) cohort by removing eight health conditions that were associated with being in less deprived socioeconomic groups. When adjusted for maternal age, ethnicity and gravidity, multimorbidity (defined by 71 health conditions) was associated with deprivation (most deprived quintile aOR $1.26,1.10$ to 1.44 ). This association was attenuated and 
Table 1 Baseline characteristics of pregnant women in CPRD (UK), SAIL (Wales) and SMR (Scotland) in 2018

\begin{tabular}{|c|c|c|c|c|c|c|}
\hline \multirow{3}{*}{$\begin{array}{l}\text { Characteristics } \\
\text { Total }\end{array}$} & \multicolumn{6}{|c|}{ Frequency (percentage) } \\
\hline & \multicolumn{2}{|l|}{ CPRD (UK) } & \multicolumn{2}{|c|}{ SAIL (Wales) } & \multicolumn{2}{|c|}{ SMR (Scotland) } \\
\hline & 37,641 & - & 27,782 & - & 6099 & - \\
\hline \multicolumn{7}{|l|}{ Nation } \\
\hline England & 13,075 & $(34.74 \%)$ & - & - & - & - \\
\hline Northern Ireland & 2984 & (7.93\%) & - & - & - & - \\
\hline Scotland & 12,559 & (33.37\%) & - & - & - & - \\
\hline Wales & 9023 & (23.97\%) & - & - & - & - \\
\hline \multicolumn{7}{|l|}{ Age categories (5 yearly) } \\
\hline $15-19$ & 2534 & $(6.73 \%)$ & 1537 & $(5.53 \%)$ & 422 & $(6.92 \%)$ \\
\hline $20-24$ & 6604 & $(17.54 \%)$ & 5360 & $(19.29 \%)$ & 1147 & $(18.81 \%)$ \\
\hline $25-29$ & 10,204 & (27.11\%) & 8617 & $(31.02 \%)$ & 1830 & $(30.00 \%)$ \\
\hline $30-34$ & 10,723 & (28.49\%) & 8081 & $(29.09 \%)$ & 1746 & $(28.63 \%)$ \\
\hline $35-39$ & 5970 & (15.86\%) & 3549 & $(12.77 \%)$ & 803 & $(13.17 \%)$ \\
\hline $40-44$ & 1428 & (3.79\%) & 603 & $(2.17 \%)$ & 138 & $(2.26 \%)$ \\
\hline $45-49$ & 178 & $(0.47 \%)$ & 35 & $(0.13 \%)$ & 13 & $(0.21 \%)$ \\
\hline \multicolumn{7}{|l|}{ Gravidity } \\
\hline 1 & 11,480 & (30.50\%) & 13,006 & $(46.81 \%)$ & 1800 & $(29.51 \%)$ \\
\hline 2 & 9895 & (26.29\%) & 9972 & (35.89\%) & 1992 & $(32.66 \%)$ \\
\hline 3 & 6734 & $(17.89 \%)$ & 3252 & (11.71\%) & 1105 & $(18.12 \%)$ \\
\hline 4 & 4004 & (10.64\%) & 1035 & $(3.73 \%)$ & 580 & $(9.51 \%)$ \\
\hline$\geq 5$ & 5528 & (14.69\%) & 517 & $(1.86 \%)$ & 618 & $(10.13 \%)$ \\
\hline Missing & - & - & - & - & 4 & $(0.07 \%)$ \\
\hline \multicolumn{7}{|l|}{ Ethnicity } \\
\hline Asian / South Asians ${ }^{\mathrm{a}}$ & 1261 & $(3.35 \%)$ & 418 & $(1.50 \%)$ & 149 & $(2.44 \%)$ \\
\hline Black & 973 & $(2.58 \%)$ & 178 & $(0.64 \%)$ & 23 & $(0.38 \%)$ \\
\hline Mixed & 305 & (0.81\%) & 121 & $(0.44 \%)$ & 8 & $(0.13 \%)$ \\
\hline Other & 528 & $(1.40 \%)$ & 229 & $(0.82 \%)$ & 91 & $(1.49 \%)$ \\
\hline White & 20,818 & (55.31\%) & 17,430 & $(62.74 \%)$ & 4903 & $(80.39 \%)$ \\
\hline Missing & 13,756 & $(36.55 \%)$ & 9406 & (33.86\%) & 925 & $(15.17 \%)$ \\
\hline \multicolumn{7}{|l|}{ BMI $\left(\mathrm{kg} / \mathrm{m}^{2}\right)$} \\
\hline Underweight $(<18.5)$ & 1217 & $(3.23 \%)$ & 1287 & $(4.63 \%)$ & 92 & $(1.51 \%)$ \\
\hline Normal Weight (18.5-24.9) & 14,440 & $(38.36 \%)$ & 9485 & $(34.14 \%)$ & 1478 & $(24.23 \%)$ \\
\hline Overweight (25-29.9) & 8075 & $(21.45 \%)$ & 5658 & $(20.37 \%)$ & 1010 & $(16.56 \%)$ \\
\hline Obese $(30+)$ & 7178 & $(19.07 \%)$ & 5372 & $(19.34 \%)$ & 1279 & $(20.97 \%)$ \\
\hline Missing & 6731 & $(17.88 \%)$ & 5980 & $(21.52 \%)$ & 2240 & $(36.73 \%)$ \\
\hline \multicolumn{7}{|l|}{ Smoking } \\
\hline Non-Smoker & 22,395 & $(59.50 \%)$ & 10,151 & $(36.54 \%)$ & 3349 & $(54.91 \%)$ \\
\hline Ex-smoker & 5707 & $(15.16 \%)$ & 8022 & $(28.87 \%)$ & 863 & $(14.15 \%)$ \\
\hline Smoker & 8237 & $(21.88 \%)$ & 6612 & $(23.80 \%)$ & 1041 & $(17.07 \%)$ \\
\hline Missing & 1302 & $(3.46 \%)$ & 2997 & $(10.79 \%)$ & 846 & $(13.87 \%)$ \\
\hline Patient level deprivation quintiles (IMD) & \multicolumn{6}{|c|}{ Only available for England ${ }^{b}$} \\
\hline 1, least deprived & 2326 & (17.79\%) & 6455 & $(23.23 \%)$ & 722 & $(11.84 \%)$ \\
\hline 2 & 1835 & (14.03\%) & 5460 & (19.65\%) & 1039 & $(17.04 \%)$ \\
\hline 3 & 1878 & $(14.36 \%)$ & 4779 & (17.20\%) & 979 & (16.05\%) \\
\hline 4 & 1853 & $(14.17 \%)$ & 4032 & (14.51\%) & 1253 & $(20.54 \%)$ \\
\hline 5 , most deprived & 1908 & (14.59\%) & 3832 & (13.79\%) & 1344 & (22.04\%) \\
\hline Missing & 3275 & $(25.05 \%)$ & 3224 & (11.60\%) & 762 & $(12.49 \%)$ \\
\hline
\end{tabular}

a South Asian for CPRD, Asian for SAIL and SMR

${ }^{\text {b }}$ Aggregate IMD quintiles cannot be provided for UK as each nation has its specific IMD; data presented here is patient level IMD for England only $(n=13,075)$. Practice level IMD for all four UK nations in CPRD is available in Additional Table 1

$B M I$ body mass index, CPRD Clinical Practice Research Datalink, IMD Index of Multiple Deprivation, SAIL The Secure Anonymized Information Linkage databank, SMR Scottish Morbidity Records 
was no longer significant when raised BMI and smoking status were added (aOR 1.08, 0.94 to 1.24, Additional Table 6).

\section{Individual health conditions}

Table 3 presents the top 20 most prevalent health conditions in our study cohort. The top four most common health conditions across all three datasets were depression, anxiety (both known as common mental health disorders), allergic rhinoconjunctivitis and asthma, with the prevalence of common mental health disorders being consistently around $20 \%$. The full list of prevalence for each health condition is presented in Additional Table 7.

\section{Combinations of multimorbidity}

Table 4 presents the top ten most common combinations of multimorbidity, the most prevalent combinations being depression and anxiety in primary care datasets (2.2 and $2.7 \%$ of pregnant women in CPRD and SAIL respectively) and common mental health disorders and asthma for SMR (3.2\%). The presented prevalence is for mutually exclusive multimorbidity combinations, and therefore prevalence for depression and anxiety will not include women with depression, anxiety and other health condition/s. When only considering physical conditions, the most common combination was asthma and allergic rhinoconjunctivitis $(1.7,2.1$ and 2.2\% in CPRD, SAIL and SMR respectively).

\section{Prevalence of multimorbidity in pregnant women with selected health conditions}

These examples have been provided to illustrate the burden of using the CPRD (UK) pregnancy cohort in 2018. The featured health conditions were the leading causes of maternal deaths in the MBRRACE-UK report [4].

Cardiovascular disease (ischemic heart disease, stroke/ transient ischemic attack, heart failure, atrial fibrillation, congenital heart disease, valvular heart disease, cardiomyopathy, hypertension) affected 2.0\% (745/37641) of pregnant women, of whom $80.1 \%(597 / 745)$ had multimorbidity. Less than $1 \%(246 / 37641)$ of pregnant women had a history of venous thromboembolism, among whom $85.8 \%(211 / 246)$ had multimorbidity. Epilepsy affected $1.4 \%$ (543/37641) pregnant women, among whom $80.7 \%$ $(438 / 543)$ had multimorbidity.

\section{Prevalence of selected health conditions by social deprivation and ethnicity}

Table 5 presents examples to illustrate the difference in the prevalence of individual health conditions by patient level social deprivation and ethnicity using CPRD (England). Mental health conditions, asthma and epilepsy increased with deprivation. In contrast, some of the common health conditions were more common in the affluent groups, including anxiety, migraine, irritable bowel syndrome, and polycystic ovarian syndrome. For ethnicity, mental health conditions, asthma, migraine, irritable bowel syndrome and psoriasis were more prevalent in White ethnic group; whilst allergic rhinoconjunctivitis and polycystic ovarian syndrome were more prevalent in ethnic minority groups.

\section{Discussion}

\section{Main findings}

This study used contemporaneous, routinely collected datasets to study the epidemiology of multimorbidity (defined as having two or more long-term physical or mental health conditions) in pregnant women in the UK. Two in five pregnant women had pre-existing multimorbidity. One in five pregnant women had multimorbidity that were active in the year before pregnancy. Seven in ten pregnant women with multimorbidity had a history of mental health condition/s. In women with conditions that are known to be leading causes of maternal death [4], four in five had pre-existing multimorbidity. Pregnant women with multimorbidity were more likely to be older, multigravid, smoked or have raised BMI preconception.

\section{Strengths and limitations}

This study utilized electronic health records which provided a rich source of data and is generalizable across different settings. It avoided misclassification bias associated with self-reported surveys. However, as with all research that use routine health records, it is subjected to residual confounding and can be limited by the quality and consistency of data entry by clinicians and administrators [14]. We have attempted to improve the accuracy of health conditions ascertainment through the design of our phenome definitions (e.g., using additional age limit and phenomes by prescriptions).

The definition of multimorbidity used in this study was based on simple counting of conditions, without weightings. There is currently no single multimorbidity index that can measure multimorbidity in all settings definitively [15]. The only currently available validated comorbidity index in maternal health research was developed using secondary care data and only included 20 conditions [16], in comparison to the 79 conditions prioritized by our multidisciplinary group and patient representatives. Obesity was analysed as a covariate (BMI categories) in this study; the prevalence of multimorbidity would be higher if obesity was considered a long-term health condition. 


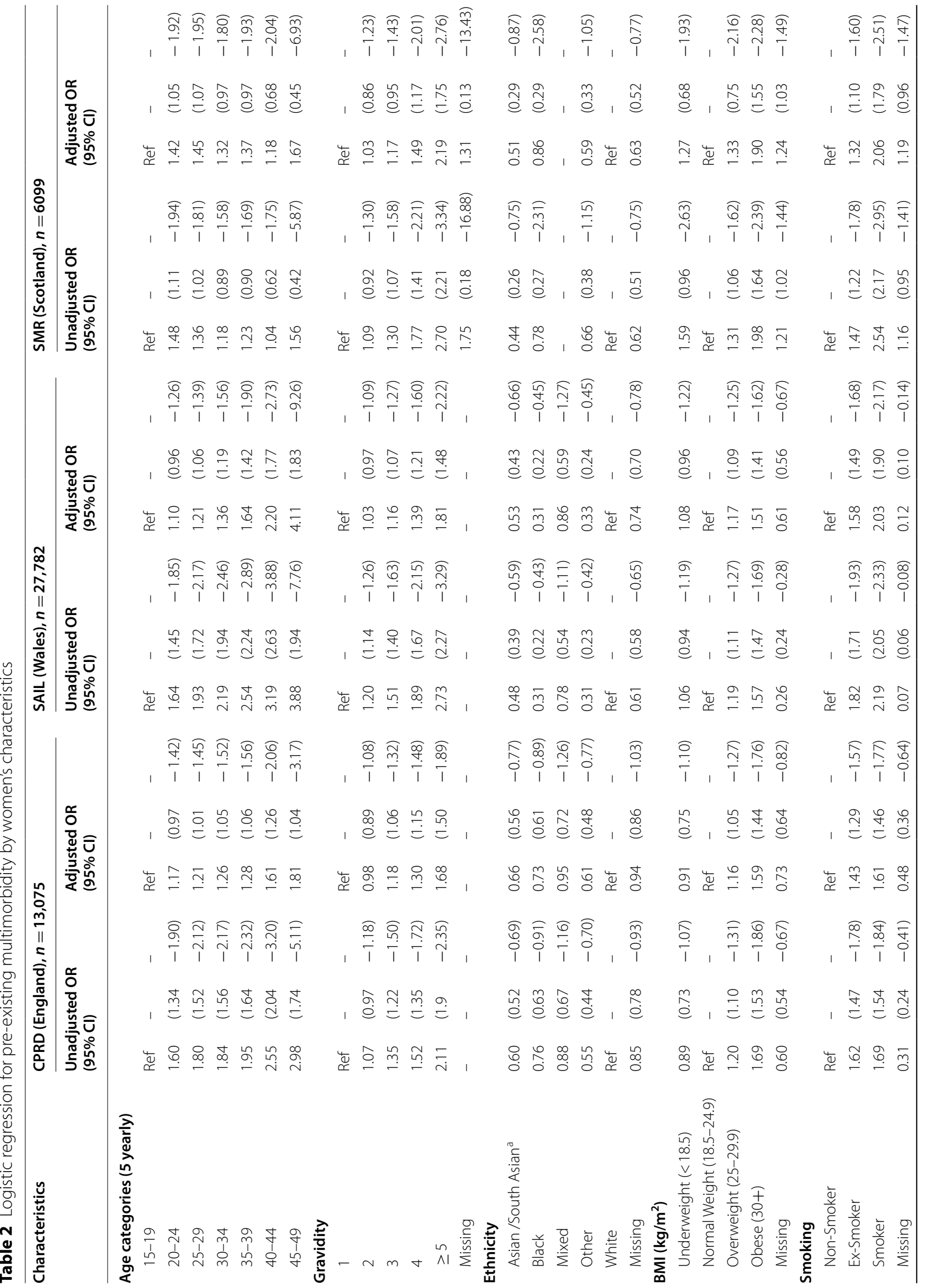




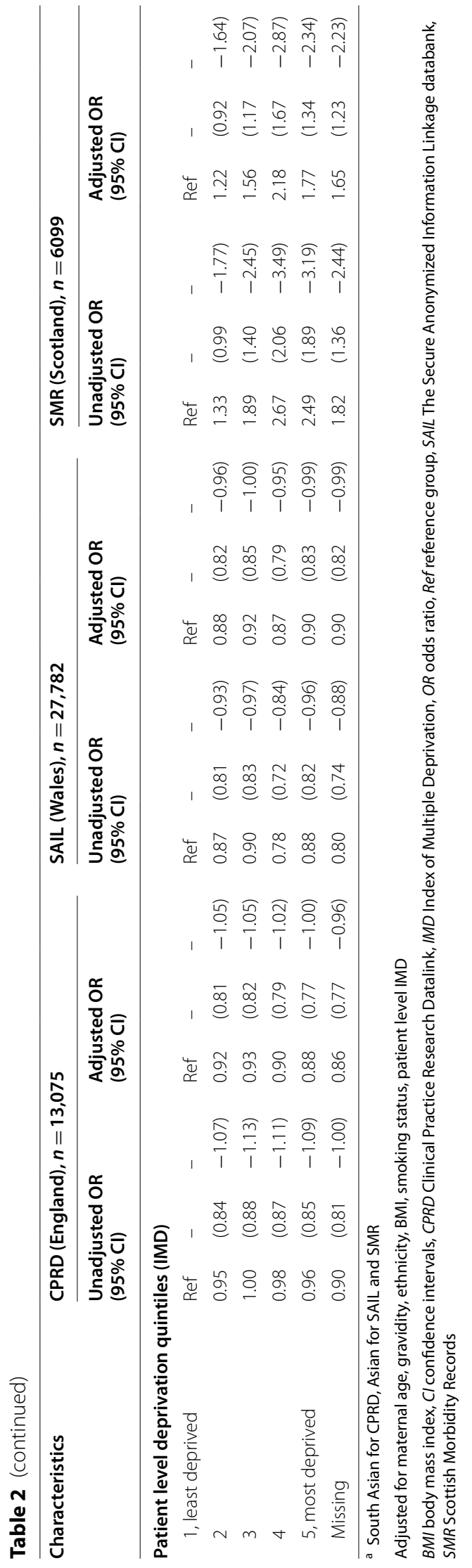




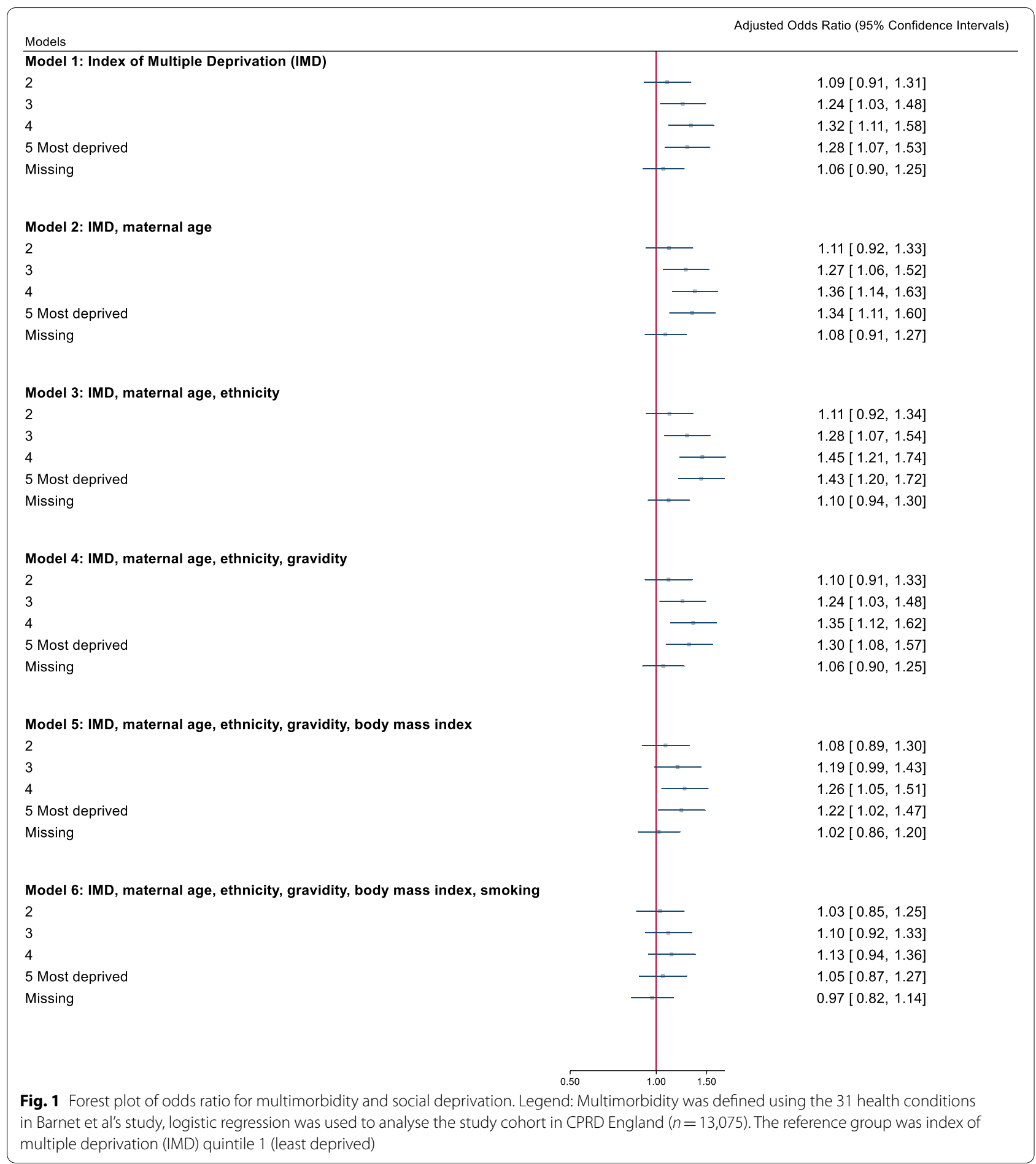

\section{Utility of the different datasets}

Compared with CPRD and SAIL primary care datasets, the prevalence of non-life-threatening health conditions such as allergic rhinoconjunctivitis, migraine, irritable bowel syndrome and, ultimately, multimorbidity was lower in the Scottish secondary care with linked community prescription dataset.

This is likely to reflect that health conditions seen in primary care encompass the whole severity spectrum. Some common conditions, such as anxiety or 


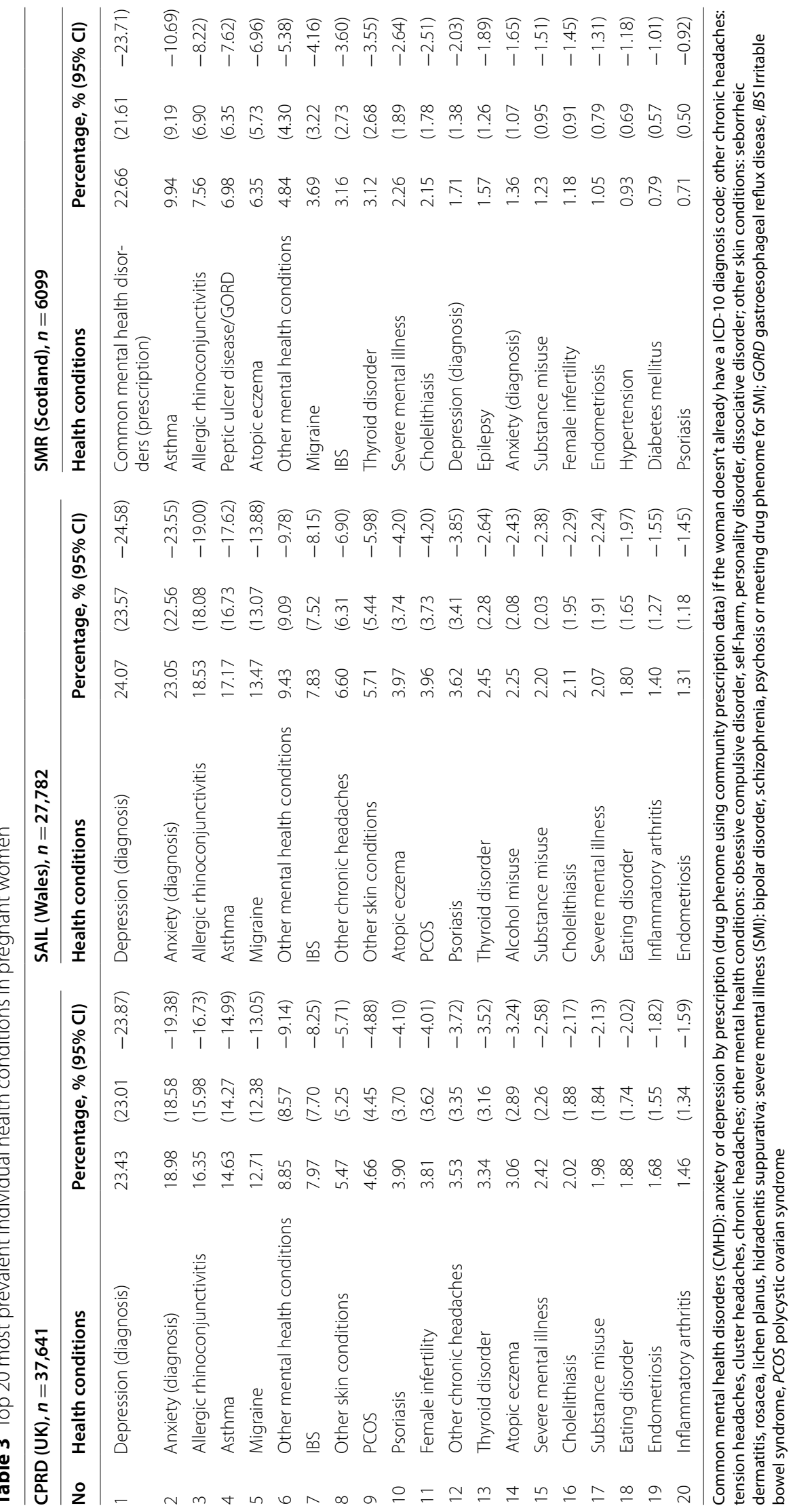


Table 4 Top ten mutually exclusive combinations of multimorbidity in pregnant women

\begin{tabular}{|c|c|c|c|c|c|c|c|c|}
\hline \multicolumn{3}{|l|}{ CPRD (UK), $n=37,641$} & \multicolumn{3}{|l|}{ SAIL (Wales), $n=27,782$} & \multicolumn{3}{|l|}{ SMR (Scotland), $n=6099$} \\
\hline All health conditions & $\mathrm{n}$ & $\%$ & All health conditions & $\mathrm{n}$ & $\%$ & All health conditions & $\mathrm{n}$ & $\%$ \\
\hline Anxiety, Depression & 825 & $2.19 \%$ & Anxiety, Depression & 748 & $2.69 \%$ & $\begin{array}{l}\text { Common mental health disorders } \\
\text { (CMHD), Asthma }\end{array}$ & 195 & $3.20 \%$ \\
\hline Asthma, Allergic rhinoconjunctivitis & 370 & $0.98 \%$ & Asthma, Allergic rhinoconjunctivitis & 319 & $1.15 \%$ & Peptic ulcer disease/GORD, CMHD & 195 & $3.20 \%$ \\
\hline $\begin{array}{l}\text { Depression, Other mental health } \\
\text { conditions }\end{array}$ & 214 & $0.57 \%$ & $\begin{array}{l}\text { Anxiety, Depression, Other mental } \\
\text { health conditions }\end{array}$ & 164 & $0.59 \%$ & Allergic rhinoconjunctivitis, CMHD & 145 & $2.38 \%$ \\
\hline $\begin{array}{l}\text { Migraine, Allergic rhinoconjunc- } \\
\text { tivitis }\end{array}$ & 178 & $0.47 \%$ & $\begin{array}{l}\text { Allergic rhinoconjunctivitis, Anxiety, } \\
\text { Depression }\end{array}$ & 140 & $0.50 \%$ & Allergic rhinoconjunctivitis, Asthma & 132 & $2.16 \%$ \\
\hline $\begin{array}{l}\text { Anxiety, Depression, Other mental } \\
\text { health conditions }\end{array}$ & 175 & $0.46 \%$ & Asthma, Anxiety, Depression & 138 & $0.50 \%$ & Atopic eczema, CMHD & 125 & $2.05 \%$ \\
\hline Asthma, Depression & 172 & $0.46 \%$ & Migraine, Anxiety, Depression & 128 & $0.46 \%$ & Migraine, CMHD & 101 & $1.66 \%$ \\
\hline $\begin{array}{l}\text { Allergic rhinoconjunctivitis, Depres- } \\
\text { sion }\end{array}$ & 171 & $0.45 \%$ & $\begin{array}{l}\text { Allergic rhinoconjunctivitis, Depres- } \\
\text { sion }\end{array}$ & 120 & $0.43 \%$ & $\begin{array}{l}\text { Other mental health conditions, } \\
\text { CMHD }\end{array}$ & 96 & $1.57 \%$ \\
\hline Migraine, Depression & 161 & $0.43 \%$ & $\begin{array}{l}\text { Migraine, Allergic rhinoconjunc- } \\
\text { tivitis }\end{array}$ & 117 & $0.42 \%$ & Asthma, atopic eczema & 94 & $1.54 \%$ \\
\hline Asthma, Anxiety, Depression & 140 & $0.37 \%$ & $\begin{array}{l}\text { Depression, Other mental health } \\
\text { conditions }\end{array}$ & 109 & $0.39 \%$ & Asthma, Peptic ulcer disease/GORD & 91 & $1.49 \%$ \\
\hline Asthma, Migraine ${ }^{a}$ & 136 & $0.36 \%$ & Asthma, Depression & 105 & $0.38 \%$ & Irritable bowel syndrome, CMHD & 90 & $1.48 \%$ \\
\hline Physical health conditions & n & $\%$ & Physical health conditions & $\mathbf{n}$ & $\%$ & Physical health conditions & $\mathrm{n}$ & $\%$ \\
\hline Asthma, Allergic rhinoconjunctivitis & 626 & $1.66 \%$ & Asthma, Allergic rhinoconjunctivitis & 594 & $2.14 \%$ & $\begin{array}{l}\text { Asthma, Allergic rhinoconjunc- } \\
\text { tivitis }\end{array}$ & 132 & $2.16 \%$ \\
\hline $\begin{array}{l}\text { Migraine, Allergic rhinoconjunc- } \\
\text { tivitis }\end{array}$ & 318 & $0.84 \%$ & $\begin{array}{l}\text { Migraine, Allergic rhinoconjunc- } \\
\text { tivitis }\end{array}$ & 239 & $0.86 \%$ & Asthma, Atopic eczema & 94 & $1.54 \%$ \\
\hline Asthma, Migraine ${ }^{a}$ & 273 & $0.73 \%$ & Asthma, Migraine & 189 & $0.68 \%$ & Asthma, Peptic ulcer disease/GORD & 91 & $1.49 \%$ \\
\hline $\begin{array}{l}\text { Allergic rhinoconjunctivitis, Irritable } \\
\text { bowel syndrome }\end{array}$ & 175 & $0.46 \%$ & $\begin{array}{l}\text { Allergic rhinoconjunctivitis, Irritable } \\
\text { bowel syndrome }\end{array}$ & 143 & $0.51 \%$ & $\begin{array}{l}\text { Irritable bowel syndrome, Peptic } \\
\text { ulcer disease/GORD }\end{array}$ & 79 & $1.30 \%$ \\
\hline Migraine, Irritable bowel syndrome & 175 & $0.46 \%$ & Migraine, Irritable bowel syndrome & 136 & $0.49 \%$ & $\begin{array}{l}\text { Allergic Rhinitis, Peptic ulcer } \\
\text { disease/GORD }\end{array}$ & 76 & $1.25 \%$ \\
\hline $\begin{array}{l}\text { Allergic rhinoconjunctivitis, Other } \\
\text { skin conditions }\end{array}$ & 140 & $0.37 \%$ & $\begin{array}{l}\text { Asthma, Migraine, Allergic rhino- } \\
\text { conjunctivitis }\end{array}$ & 129 & $0.46 \%$ & $\begin{array}{l}\text { Atopic eczema, Peptic ulcer dis- } \\
\text { ease/GORD }\end{array}$ & 64 & $1.05 \%$ \\
\hline $\begin{array}{l}\text { Asthma, Migraine, Allergic rhino- } \\
\text { conjunctivitis }\end{array}$ & 133 & $0.35 \%$ & Migraine, Other chronic headaches & 123 & $0.44 \%$ & $\begin{array}{l}\text { Allergic rhinoconjunctivitis, Atopic } \\
\text { eczema }\end{array}$ & 62 & $1.02 \%$ \\
\hline Asthma, Irritable bowel syndrome & 117 & $0.31 \%$ & $\begin{array}{l}\text { Allergic rhinoconjunctivitis, Other } \\
\text { chronic headaches }\end{array}$ & 115 & $0.41 \%$ & $\begin{array}{l}\text { Migraine, Peptic ulcer disease/ } \\
\text { GORD }\end{array}$ & 50 & $0.82 \%$ \\
\hline Migraine, Other skin conditions & 98 & $0.26 \%$ & Asthma, Irritable bowel syndrome & 110 & $0.40 \%$ & Asthma, Irritable bowel syndrome & 45 & $0.74 \%$ \\
\hline $\begin{array}{l}\text { Allergic rhinoconjunctivitis, Other } \\
\text { chronic headaches }\end{array}$ & 87 & $0.23 \%$ & $\begin{array}{l}\text { Allergic rhinoconjunctivitis, Other } \\
\text { skin conditions }\end{array}$ & 98 & $0.35 \%$ & $\begin{array}{l}\text { Cholelithiasis, Peptic ulcer disease/ } \\
\text { GORD }\end{array}$ & 43 & $0.71 \%$ \\
\hline
\end{tabular}

These multimorbidity combinations are mutually exclusive. For instance, the count for 'anxiety and depression' will include women with exactly these two conditions only, it does not include women with combinations of 'anxiety, depression' and other condition/s

a The percentage of asthma and migraine multimorbidity combination is higher when considering physical health conditions only as it would include combination of these conditions with mental health conditions which are no longer accounted for

GORD Gastroesophageal reflux disease

depression, may only present to primary care, some of which are managed conservatively without prescribed medications. In contrast, the Scottish secondary care and community prescription database would only capture the severe spectrum of a condition that requires hospital attendance or regular prescriptions and may under-estimate the prevalence of multimorbidity. This confirms that primary care health records may be a more comprehensive data source to study pre-existing multimorbidity in pregnant women, and to identify the target at risk population for preconception intervention.

Similar findings were observed in both CPRD, and SAIL add to the validity of these findings. Whilst CPRD offered the benefit of representing data from all four UK nations, SAIL offered a more complete coverage at a population level in Wales with good follow up throughout an individual's lifetime even when they change GP practices.

Our study highlighted a shortfall in the recording of ethnicity and preconception body mass index, and to a 
Table 5 Prevalence of selected health conditions in pregnant women by social deprivation and ethnicity

\begin{tabular}{|c|c|c|c|c|c|c|c|}
\hline \multirow{3}{*}{ Health conditions } & \multicolumn{6}{|c|}{$\%$ by patient level IMD quintiles in CPRD (England), $n=13,075$} & \multirow[t]{3}{*}{$P$ value for $X^{2}$ test } \\
\hline & 1 , least deprived & 2 & 3 & 4 & 5 , most deprived & Missing & \\
\hline & $n=2326$ & $n=1835$ & $n=1878$ & $n=1853$ & $n=1908$ & $n=3275$ & \\
\hline \multicolumn{8}{|c|}{ Example of health conditions that increased with deprivation } \\
\hline${ }^{* * *}$ Depression (diagnosis) & 20.55 & 22.29 & 24.55 & 24.82 & 25.58 & 22.32 & $<0.001$ \\
\hline *Asthma & 12.85 & 14.22 & 14.70 & 15.00 & 14.83 & 12.61 & 0.049 \\
\hline${ }^{* * *}$ Other mental health conditions & 5.33 & 6.21 & 8.31 & 9.01 & 10.01 & 6.93 & $<0.001$ \\
\hline Psoriasis & 3.31 & 4.31 & 3.09 & 4.16 & 3.41 & 3.82 & 0.258 \\
\hline Cardiovascular disease & 2.06 & 2.07 & 1.70 & 1.89 & 2.67 & 2.29 & 0.378 \\
\hline ***Severe mental illness & 1.16 & 1.58 & 1.60 & 2.75 & 2.99 & 2.05 & $<0.001$ \\
\hline${ }^{* *}$ Epilepsy & 0.90 & 1.20 & 1.65 & 2.00 & 2.04 & 1.04 & 0.002 \\
\hline Venous thromboembolism & 0.77 & 0.65 & 0.59 & 0.49 & 1.26 & 0.98 & 0.073 \\
\hline **Substance misuse & 0.52 & 1.36 & 1.33 & 1.46 & 2.10 & 1.37 & 0.001 \\
\hline \multicolumn{8}{|c|}{ Example of health conditions that decreased with deprivation } \\
\hline${ }^{* *}$ Anxiety (diagnosis) & 20.77 & 20.05 & 21.35 & 18.78 & 17.82 & 17.77 & 0.005 \\
\hline Allergic rhinoconjunctivitis & 18.14 & 16.13 & 16.03 & 16.68 & 15.57 & 16.15 & 0.254 \\
\hline${ }^{*}$ Migraine & 14.32 & 13.35 & 15.44 & 12.14 & 13.16 & 12.76 & 0.034 \\
\hline **|rritable bowel syndrome & 10.15 & 8.88 & 9.16 & 7.99 & 6.71 & 9.04 & 0.003 \\
\hline **Polycystic ovarian syndrome & 8.34 & 5.78 & 7.03 & 6.26 & 5.29 & 6.41 & 0.001 \\
\hline Other skin conditions & 6.58 & 6.70 & 6.02 & 6.80 & 4.98 & 6.44 & 0.179 \\
\hline \multirow[t]{2}{*}{ Alcohol misuse } & 0.82 & 0.65 & 0.59 & 0.81 & 0.68 & 0.55 & 0.820 \\
\hline & \multicolumn{6}{|c|}{ \% by ethnicity in CPRD (England) } & \multirow[t]{3}{*}{$P$ value for $X^{2}$ test } \\
\hline \multirow[t]{2}{*}{ Health conditions } & White & Black & Mixed & Others & South Asians & Missing & \\
\hline & $n=8302$ & $n=490$ & $n=214$ & $n=336$ & $n=843$ & $n=2890$ & \\
\hline \multicolumn{8}{|c|}{ Example of health conditions that were more prevalent in White ethnic group } \\
\hline ***Depression (diagnosis) & 25.20 & 13.27 & 20.56 & 14.29 & 10.32 & 23.91 & $<0.001$ \\
\hline ***Anxiety (diagnosis) & 21.27 & 9.80 & 20.09 & 10.42 & 10.08 & 18.86 & $<0.001$ \\
\hline${ }^{* * *}$ Asthma & 14.33 & 11.22 & 14.02 & 6.55 & 10.79 & 14.60 & $<0.001$ \\
\hline ***Migraine & 14.30 & 10.20 & 11.21 & 8.63 & 9.85 & 13.46 & $<0.001$ \\
\hline ***|rritable bowel syndrome & 10.07 & 9.80 & 6.07 & 2.68 & 3.91 & 7.06 & $<0.001$ \\
\hline${ }^{* * *}$ Other mental health conditions & 8.44 & 5.31 & 10.28 & 4.17 & 1.90 & 6.92 & $<0.001$ \\
\hline Other skin conditions & 6.75 & 3.88 & 5.61 & 5.06 & 5.93 & 5.64 & 0.050 \\
\hline${ }^{* * *}$ Psoriasis & 4.05 & 1.22 & 3.27 & 1.49 & 1.66 & 3.91 & $<0.001$ \\
\hline Serious mental illness & 2.11 & 1.63 & 1.40 & 0.60 & 1.19 & 2.18 & 0.159 \\
\hline **Substance misuse & 1.47 & 0.82 & 1.40 & 0.00 & 0.00 & 1.56 & 0.002 \\
\hline Epilepsy & 1.46 & 0.41 & 2.80 & 0.60 & 1.07 & 1.52 & 0.099 \\
\hline Venous thromboembolism & 0.93 & 0.41 & 1.40 & 0.60 & 0.47 & 0.62 & 0.309 \\
\hline${ }^{* * *}$ Alcohol misuse & 0.90 & 0.00 & 1.40 & 0.30 & 0.00 & 0.31 & $<0.001$ \\
\hline \multicolumn{8}{|c|}{ Example of health conditions that were more prevalent in ethnic minority groups } \\
\hline${ }^{* * *}$ Allergic rhinoconjunctivitis & 15.56 & 22.65 & 17.29 & 19.94 & 16.25 & 17.65 & $<0.001$ \\
\hline ***Polycystic ovarian syndrome & 6.60 & 5.10 & 8.88 & 8.93 & 9.85 & 5.33 & $<0.001$ \\
\hline Cardiovascular disease & 2.18 & 3.88 & 1.40 & 1.79 & 2.14 & 1.80 & 0.090 \\
\hline
\end{tabular}

The selected health conditions were the top ten most common conditions in this study or leading causes of maternal death. Other mental health conditions: obsessive compulsive disorder, self-harm, personality disorder, dissociative disorder. Severe mental illness (SMI): bipolar disorder, schizophrenia, psychosis or meeting drug phenome for SMI. Other skin conditions: seborrheic dermatitis, rosacea, lichen planus, hidradenitis suppurativa

${ }^{*}$ denotes $p<0.05,{ }^{* *}$ denotes $p<0.01,{ }^{* * *}$ denotes $p<0.001$

lesser extent, smoking status preconception for pregnant women in routine health records. Patient level data for social deprivation was limited by the availability of data linkage in CPRD. Although sensitivity analysis in the
CPRD (England) dataset with imputed ethnicity and IMD showed similar findings with the primary analysis, the interpretation of the association analysis should be taken with caution. In SAIL, pregnancy episodes were detected 
from the National Community Child Health database (NCCHD), and this does not include pregnancies that resulted in early pregnancy loss; hence the gravidity data generated from this database is likely to be an underestimation. Historical data from the SMR datasets were available from 2005 to 2019. This meant that if a pregnant woman had a history of a health condition prior to this time period, it may not be captured. This limitation is more likely to affect older women in the SMR pregnancy cohort and may partially account for the lack of association of maternal age with multimorbidity. Further limitations of each dataset are outlined in Additional file 5.

\section{Results in the context of what is known \\ High prevalence of multimorbidity in pregnant women}

The current evidence for the prevalence of multimorbidity in pregnant women is scarce and findings vary widely. This ranged between $<10$ to $35 \%[2,3,17,18]$. The high prevalence of multimorbidity in pregnant women in this study is concerning as it is associated with adverse outcomes for mother and child [2-4]. In the latest MBRRACE UK maternal mortality enquiry report, $90 \%$ of maternal deaths up to one year post pregnancy occurred in women with multiple health and social problems [4]. MBRRACE has called for national guidance for the management of pregnant women with multiple morbidities and social factors [19]. Recently, the Ockenden report (a high profile UK independent inquiry of maternity services at a local hospital) highlighted the need for involvement of maternal medicine specialist and maternal mental health services for managing women with complex pregnancies [20].

\section{Clinical implications}

Our study provided a current snapshot of how multimorbidity is distributed in the UK in terms of sociodemographics, the health conditions that constitute multimorbidity and the common combinations of health conditions in pregnant women. Mental health conditions were particularly prevalent and contributed to $70 \%$ of multimorbidity in pregnant women. Psychiatric causes were amongst the leading cause of maternal death in the UK [4]. Our findings further support the need for integration of mental health services with maternity services and equitable access to perinatal mental health services in the UK [21].

\section{Social deprivation, ethnicity and multimorbidity in pregnant women}

Post hoc analysis found that some health conditions were more prevalent in affluent pregnant women (e.g., anxiety, irritable bowel syndrome), potentially masking the association of multimorbidity with social deprivation. When these conditions were removed, multimorbidity was associated with social deprivation but this effect was not observed when BMI and smoking status were also adjusted for. This suggests that smoking and obesity may mediate the relationship between social deprivation and multimorbidity in pregnant women.

Many of the topmost common health conditions were more prevalent in White pregnant women, particularly mental health conditions. This may have contributed to the lack of association of ethnic minority groups with multimorbidity. Previous literature reported that people of ethnic minority are less likely to access/receive mental health support/treatment $[22,23]$. In addition, stigma associated with mental health conditions was reported to be higher in ethnic minorities [24].

Both observations mean that there could be health care access issues for some of the common health conditions, especially mental health conditions, for people from socially deprived and ethnic minority groups prior to pregnancy. In addition, it strengthens the importance of addressing smoking and obesity preconception especially in pregnant women with multimorbidity from socially deprived groups. Smoking and obesity are two well-known modifiable risk factors for adverse pregnancy outcomes $[25,26]$, the added impact of multimorbidity is likely to compound this. Further research is required to quantify this, but interventions addressing smoking and obesity may help reduce adverse outcomes in pregnant women with multimorbidity.

\section{Research implications}

Despite the high prevalence of multimorbidity in pregnant women, and the associated adverse outcomes, there is currently a paucity of evidence in this field. The MuM-PreDiCT consortium is a multidisciplinary collaboration across all four nations in the UK, including women with lived experience of multimorbidity and pregnancy. Our next step is to quantify the impact of multimorbidity on pregnancy, maternal and offspring outcomes. This will provide crucial information for women with multimorbidity who are planning a pregnancy and results from the outcome studies may require us to reconsider how we categorize high-risk pregnancy. The ultimate aim is to produce high quality evidence that would guide clinical practice to prevent pregnancy complications and to optimize long-term maternal and offspring health for pregnant women with multimorbidity. 


\section{Conclusion}

A significant proportion of women enter pregnancy with pre-existing multimorbidity, especially with mental health condition/s. Amongst pregnant women with health conditions known to be leading causes of maternal death, prevalence of multimorbidity was high. Pregnant women with multimorbidity were more likely to smoke and have a raised BMI and support maybe required to address this. There may be health care access inequalities for some health conditions, especially mental health conditions in pregnant women from deprived or ethnic minority groups.

\section{Abbreviations}

BMI: Body mass index; CMHD: Common mental health disorder; CPRD: Clinical Practice Research Datalink; GORD: Gastroesophageal reflux disease; GP: General practitioner; HIC: Health Informatics Centre; IBS: Irritable bowel syndrome; IMD: Index of Multiple Deprivation; NCCHD: National Community Child Health database; NHS: National Health Service; OR: Odds ratio; PCOS: Polycystic ovarian syndrome; PPI: Patient and Public Involvement; Ref: Reference; SAIL: Secure Anonymized Information Linkage; SMI: Severe mental illness; SMR: Scottish Morbidity Records; SOP: Standard Operating Procedures; UK: United Kingdom.

\section{Supplementary Information}

The online version contains supplementary material available at https://doi. org/10.1186/s12884-022-04442-3.

Additional file 1. Cohort selection and data quality checks.

Additional file 2. Read codes and International Classification of Diseaseversion 10 (ICD-10) codes for health conditions.

Additional file 3. Phenome definitions of health conditions.

Additional file 4. The RECORD statement.

Additional file 5. Limitations of CPRD, SAIL, SMR.

Additional file 6: Figure 1. Flow chart for selection of study population.

Additional file 7: Table 1. Practice level index of multiple deprivation (IMD) quintile by nations in the CPRD 2018 pregnancy cohort.

Additional file 8: Table 2. Percentage of pregnant women by the total morbidity count in CPRD, SAIL, SMR in 2018.

Additional file 9: Table 3. Prevalence of pre-existing multimorbidity in pregnant women in CPRD, SAIL, SMR in 2018 by women's characteristics.

Additional file 10: Table 4. Sensitivity analysis of CPRD England study cohort $(n=13,075)$ with imputed ethnicity and deprivation data.

Additional file 11: Table 5. Post hoc logistic regression with multimorbidity defined using list of 31 conditions from Barnet et al's paper.

Additional file 12: Table 6. Post hoc logistic regression removing conditions that were associated with less deprived IMD quintiles in CPRD England study cohort $(n=13,075)$.

Additional file 13: Table 7. Prevalence of individual health conditions in pregnant women aged 15-49years in CPRD, SAIL, SMR in 2018.

\section{Acknowledgements}

We would like to thank our patient and public involvement representatives for their input with this study; the academic clinicians in the THINKING group for creating the Read Code lists that are not available from the literature; Professor Helen Dolk (Ulster University) for her feedback on the manuscript and Krishna Margadhamane Gokhale and Alecsandru Vitoc (University of Birmingham) for data extraction.
Patient involvement

Our patient representatives comprised of two patient and public (PPI) coinvestigators and a PPI advisory group of six women with lived experience of multimorbidity and pregnancy, with various long-term conditions. They were involved with selecting the 79 long-term health conditions used to define multimorbidity. They were also involved in interpreting the results, in particular, they noted that prevalent conditions may be on the milder spectrum and do not necessarily require specialist antenatal care. This led to additional analysis on health conditions that were leading causes of maternal death in the MBRRACE report [4]. Their feedback shaped how we presented and disseminated our findings to the public, including choosing the terminology of describing multimorbidity that they were comfortable with (two or more long-term conditions). Finally, our PPI co-investigators contributed to the preparation of this manuscript.

\section{Authors' contributions}

Our authors list includes PPI co-investigators NM and RP. SIL, AAL, UA, JIK, HH, AS, AA, BT, CNP, CY, KAE, NM, PB, RP, ST, MB, DOR, KMA, SB, KN, CM conceived the study, identified the list of health conditions to define multimorbidity and defined the phenome; SB, JIK, CM, AAL, UA, KN, SIL, AS acquired the data and required approval; SIL led the data analysis and drafting of the manuscript with contribution from AS, AAL, UA, JIK, AFF, FC; AS, AA, KN, SIL, HH curated the Read codes, UA and AAL curated the ICD-10 codes; SIL, AAL, UA, JIK, AFF, HH, $A S, A A, B T, C N P, C D M, C Y, F C, G S, K A E$, ZV, ML, NM, PB, RP, ST, MB, DOR, KMA, $S B, K N, C M$ contributed to the interpretation of the data. $A A L, U A, J I K, A F F, H H$, $A S, A A, B T, C N P, C D M, C Y, F C, G S, K A E, Z V, M L, N M, P B, R P, S T, M B, D O R, K M A$, $\mathrm{SB}, \mathrm{KN}, \mathrm{CM}$ critically revised the manuscript for important intellectual content; all authors approved the final version of the manuscript and agree to be accountable for all aspects of the work.

\section{Funding}

This work was funded by the Strategic Priority Fund "Tackling multimorbidity at scale" programme (grant number MR/W014432/1) delivered by the Medical Research Council and the National Institute for Health Research in partnership with the Economic and Social Research Council and in collaboration with the Engineering and Physical Sciences Research Council. BT was funded by the National Institute for Health Research (NIHR) West Midlands Applied Research Collaboration. AA and SIL were funded as NIHR Academic Clinical Fellows. The views expressed are those of the author and not necessarily those of the funders, the NIHR or the UK Department of Health and Social Care. The funders had no role in study design, data collection and analysis, decision to publish, or preparation of the manuscript.

\section{Availability of data and materials}

The data that support the findings of this study are available from CPRD, SAIL and the HIC at the University of Dundee but restrictions apply to the availability of these data, which were used under license for the current study, and so are not publicly available.

\section{Declarations}

\section{Ethics approval and consent to participate}

CPRD has ethics approval from the Health Research Authority to support research using anonymised patient data. The study has been approved by the Independent Scientific Advisory Committee for CPRD (reference: 20_181R) and by SAIL Information Governance Review Panel for SAIL databank. For SMR, the Health Informatics Centre (HIC) at the University of Dundee provided a linked dataset within a Safe Haven environment for this study. Dataset was obtained under HIC Standard Operating Procedures (SOP). NHS Tayside Research Ethics Committee have approved these SOPs (18/ES/0126). The School of Medicine Ethics Committee, acting on behalf of the University of St Andrews Teaching and Research Ethics Committee approved this project for SMR. As the study data are de-identified, consent is not required. All methods were performed in accordance with the guidelines and regulations as stipulated by the respective data providers.

\section{Consent for publication}

As the study data are de-identified, consent for publication is not required. 


\section{Competing interests}

None declared.

\section{Author details}

${ }^{1}$ Institute of Applied Health Research, IOEM Building, University of Birmingham, Edgbaston, Birmingham B15 2TT, UK. ${ }^{2}$ Division of Population and Behavioural Sciences, School of Medicine, University of St Andrews, St Andrews, UK. ${ }^{3}$ Data Science, Medical School, Swansea University, Swansea, UK. ${ }^{4}$ Department of Epidemiology and Medical Statistics, College of Medicine, University of Ibadan, Ibadan, Nigeria. ${ }^{5}$ Centre for Women's Mental Health, Division of Psychology and Mental Health, School of Health Sciences, Faculty of Biology Medicine \& Health, The University of Manchester, Manchester, UK. 'Guy's and St. Thomas' NHS Foundation Trust, London, UK. ${ }^{7}$ Medical and Clinical Pharmacology, School of Medicine, Université Toulouse III, Toulouse, France. ${ }^{8}$ INSERM, Centre for Epidemiology and Research in Population Health (CERPOP), CIC 1436 Toulouse, France. ${ }^{9}$ Division of Informatics, Imaging and Data Sciences, Faculty of Biology Medicine and Health, The University of Manchester, Manchester, UK. ${ }^{10}$ Health Data Research, London, UK. ${ }^{11}$ Bradford Institute for Health Research, Bradford, UK. ${ }^{12}$ Centre for Public Health, Queen's University of Belfast, Belfast, UK. ${ }^{13}$ St Michael's Hospital, University Hospitals Bristol NHS Foundation Trust, Bristol, UK. ${ }^{14}$ The Institute of Nursing and Health Research, Ulster University, Newtownabbey, UK. ${ }^{15}$ Patient and Public Representative, London, UK. ${ }^{16}$ WHO Collaborating Centre for Global Women's Health, Institute of Metabolism and Systems Research, University of Birmingham, Birmingham, UK. ${ }^{17}$ Department of Obstetrics and Gynaecology, Birmingham Women's and Children's NHS Foundation Trust, Birmingham, UK.

${ }^{18}$ Aberdeen Centre for Women's Health Research, School of Medicine, Medical Science and Nutrition, University of Aberdeen, Aberdeen, UK. ${ }^{19}$ Greater Manchester Mental Health NHS Foundation Trust, Manchester, UK.

Received: 8 November 2021 Accepted: 24 January 2022

Published online: 11 February 2022

\section{References}

1. The Academy of Medical Science. Multimorbidity: a priority for global health challenge 2018 [cited 2020 2nd Dec]. Available from: https:// acmedsci.ac.uk/file-download/82222577

2. Admon LK, Winkelman TNA, Heisler M, Dalton VK. Obstetric outcomes and delivery-related health care utilization and costs among pregnant women with multiple chronic conditions. Prev Chronic Dis. 2018;15:E21.

3. D'Arcy R, Knight M, Mackillop L. A retrospective audit of the sociodemographic characteristics and pregnancy outcomes for all women with multiple medical problems giving birth at a tertiary hospital in the UK in 2016. BJOG Int J Obstet Gynaecol. 2019;126:128.

4. Knight M, Bunch K, Tuffnell D, Shakespeare J, Kotnis R, Kenyon S, et al. Saving Lives, Improving Mothers' Care - Lessons learned to inform maternity care from the UK and Ireland Confidential Enquiries into Maternal Deaths and Morbidity 2015-17 Oxford: National Perinatal Epidemiology Unit, University of Oxford; 2019 [cited 2020 31st December]. Available from: https://www.npeu.ox.ac.uk/assets/downloads/mbrra ce-uk/reports/MBRRACE-UK\%20Maternal\%20Report\%202019\%20-\% 20WEB\%20VERSION.pdf.

5. Minassian C, Williams R, Meeraus WH, Smeeth L, Campbell OMR, Thomas SL. Methods to generate and validate a pregnancy register in the UK clinical practice research Datalink primary care database. Pharmacoepidemiol Drug Saf. 2019:28(7):923-33.

6. Herrett E, Gallagher AM, Bhaskaran K, Forbes H, Mathur R, van Staa T, et al. Data resource profile: clinical practice research Datalink (CPRD). Int J Epidemiol. 2015:44(3):827-36.

7. SAIL Databank. 10 years of spearheading data privacy and research utility [cited 2021 16th February]. Available from: https://saildatabank com/wp-content/uploads/SAIL_10_year_anniversary_brochure.pdf.

8. University of Dundee. Health Informatics Centre - Trusted Research Environment [cited 2021 4th February]. Available from: https://www. dundee.ac.uk/hic/.

9. Kuan V, Denaxas S, Gonzalez-Izquierdo A, Direk K, Bhatti O, Husain S, et al. A chronological map of 308 physical and mental health conditions from 4 million individuals in the English national health service. Lancet Digit Health. 2019;1(2):e63-77.

10. Ho IS, Azcoaga-Lorenzo A, Akbari A, Black C, Davies J, Hodgins P, et al. Examining variation in the measurement of multimorbidity in research: a systematic review of 566 studies. Lancet Public Health. 2021;6(8):e587-e97.

11. Booth N. What are the read codes? Health Libr Rev. 1994:11(3):177-82.

12. Khan NF, Harrison SE, Rose PW. Validity of diagnostic coding within the general practice research database: a systematic review. Br J Gen Pract. 2010;60(572):e128-e36.

13. Barnett K, Mercer SW, Norbury M, Watt G, Wyke S, Guthrie B. Epidemiology of multimorbidity and implications for health care, research, and medical education: a cross-sectional study. Lancet (London, England). 2012;380(9836):37-43

14. Bradley SH, Lawrence NR, Carder P. Using primary care data for health research in England - an overview. Future Healthc J. 2018;5(3):207-12.

15. Stirland LE, González-Saavedra L, Mullin DS, Ritchie CW, Muniz-Terrera G, Russ TC. Measuring multimorbidity beyond counting diseases: systematic review of community and population studies and guide to index choice. BMJ. 2020:368:m160.

16. Aoyama K, D'Souza R, Inada E, Lapinsky SE, Fowler RA. Measurement properties of comorbidity indices in maternal health research: a systematic review. BMC Pregnancy Childbirth. 2017;17(1):372.

17. Kersten I, Lange AE, Haas JP, Fusch C, Lode H, Hoffmann W, et al. Chronic diseases in pregnant women: prevalence and birth outcomes based on the SNiP-study. BMC Pregnancy Childbirth. 2014;14:75.

18. McCauley M, Zafar S, van den Broek N. Maternal multimorbidity during pregnancy and after childbirth in women in low- and middle-income countries: a systematic literature review. BMC Pregnancy Childbirth. 2020;20(1):637.

19. Knight M, Tuffnell D, Kenyon S, Shakespeare J, Gray R, Kurinczuk JJ, et al. Saving Lives, Improving Mothers' Care - Surveillance of maternal deaths in the UK 2011-13 and lessons learned to inform maternity care from the UK and Ireland Confidential Enquiries into Maternal Deaths and Morbidity 2009-13 Oxford: National Perinatal Epidemiology Unit, University of Oxford; 2015 [cited 2020 3rd July]. Available from: https:// maternalmentalhealthalliance.org/wp-content/uploads/MBRRACE-UKMaternal-Report-2015-3.pdf.

20. Department of Health and Social Care. Independent report: Ockenden review of maternity services at Shrewsbury and Telford Hospital NHS Trust 2020 [cited 2021 May 12]. Available from: https://www.gov.uk/ government/publications/ockenden-review-of-maternity-services-atshrewsbury-and-telford-hospital-nhs-trust.

21. Bauer A, Parsonage $M$, Knapp M, lemmi V, Adelaja B. The costs of perinatal mental health problems. London: LSE \& Centre for Mental Health; [cited 202031 st December]. Available from: https://www.nwcscnsena te.nhs.uk/files/3914/7030/1256/Costs_of_perinatal_mh.pdf.

22. Bignall T, Jeraj S, Helsby E, Butt J. Racial disparities in mental health: Literature and evidence review 2019 [cited 2021 May 10]. Available from: https://raceequalityfoundation.org.uk/wp-content/uploads/2020/03/ mental-health-report-v5-2.pdf.

23. NHS Digital. Adult Psychiatric Morbidity Survey: Survey of Mental Health and Wellbeing, England, 2014 [cited 2020 31st December]. Available from: https://webarchive.nationalarchives.gov.uk/20180 328140249/http://digital.nhs.uk/catalogue/PUB21748.

24. Eylem O, de Wit L, van Straten A, Steubl L, Melissourgaki Z, Danışman GT, et al. Stigma for common mental disorders in racial minorities and majorities a systematic review and meta-analysis. BMC Public Health. 2020;20(1):879.

25. Avşar TS, McLeod H, Jackson L. Health outcomes of smoking during pregnancy and the postpartum period: an umbrella review. BMC Pregnancy Childbirth. 2021;21(1):254.

26. Relph S, NMPA Project Team. NHS maternity Care for Women with a body mass index of $30 \mathrm{~kg} / \mathrm{m} 2$ or above: births between 1 April 2015 and 31 March 2017 in England, Wales and Scotland. London: RCOG; 2021.

\section{Publisher's Note}

Springer Nature remains neutral with regard to jurisdictional claims in published maps and institutional affiliations. 